\title{
Application of the SES Framework for Model-based Analysis of the Dynamics of Social-Ecological Systems
}

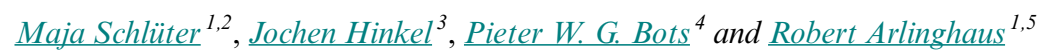

\begin{abstract}
Social-ecological systems (SES) are dynamic systems that continuously change in response to internal or external pressures. A better understanding of the interactions of the social and ecological systems that drive those dynamics is crucial for the development of sustainable management strategies. Dynamic models can serve as tools to explore social-ecological interactions; however, the complexity of the studied systems and the need to integrate knowledge, theories, and approaches from different disciplines pose considerable challenges for their development. We assess the potential of Ostrom's general SES framework (SESF) to guide a systematic and transparent process of model development in light of these difficulties. We develop a stepwise procedure for applying SESF to identify variables and their relationships relevant for an analysis of the SES. In doing so we demonstrate how the hierarchy of concepts in SESF and the identification of social-ecological processes using the newly introduced process relationships can help to unpack the system in a systematic and transparent way. We test the procedure by applying it to develop a dynamic model of decision making in the management of recreational fisheries. The added value of the common framework lies in the guidance it provides for (1) a structured approach to identifying major variables and the level of detail needed, and (2) a procedure that enhances model transparency by making explicit underlying assumptions and choices made when selecting variables and their interactions as well as the theories or empirical evidence on which they are based. Both aspects are of great relevance when dealing with the complexity of SES and integrating conceptual backgrounds from different disciplines. We discuss the advantages and difficulties of the application of SESF for model development, and contribute to its further refinement.
\end{abstract}

Key Words: dynamic modeling; model development; SES framework; social-ecological modeling; social-ecological processes

\section{INTRODUCTION}

Social-ecological systems (SES) are dynamic systems that are continuously changing (Berkes and Folke 1998, Scheffer et al. 2009). They coevolve through interactions between actors, institutions, and resources constrained and shaped by a given social-ecological setting (Holling and Gunderson 2002). Understanding coevolutionary dynamics and the way they influence the capacity of the system to cope with and adapt to global change is important for the development of sustainable management strategies (Rammel et al. 2007, Fulton et al. 2011). However, despite the importance of the dynamics of SES for their sustainability, we still lack basic understanding of them (Carpenter et al. 2009).

Dynamic models are useful tools to study the change of SES over time in situations for which time-series data are not available and experimentation difficult (Carpenter and Brock 2004). A dynamic model is defined here as a formal, theory- or empirically based, simplified mechanistic representation of the structure and processes of a real-world entity considered relevant to answer a specific question about the development of the system over time. By mathematical analysis or through simulating interactions between the social and ecological systems, dynamic models facilitate the exploration of the consequences of salient socialecological feedbacks for management and sustainability. Such models of SES can best be understood as thought experiments for hypothesis generation and testing, particularly for exploring potential future development paths of a system under a given set of assumptions and potential pressures. Their usefulness for understanding the dynamics of SES is highest when they are part of a larger process of empirical and theoretical SES research (Baumgärtner et al. 2008).
The development of SES models, however, poses many challenges. A model of the coupled dynamics of a particular real or hypothetical SES has to integrate knowledge on variables and their relationships from both the social and the ecological domains. This knowledge is scattered across many disciplines and thus difficult for a single researcher or a small disciplinary team to comprehend fully. SES models have therefore often been developed from the perspective of a single discipline, such as resource economics, applied ecology, or fisheries science (Schlüter et al. 2012). Based on the respective disciplinary frameworks and theories such approaches tend to oversimplify either the ecological or the social domain, and often fall short in exploring and explaining the social-ecological feedbacks that drive the development of the coupled SES (Schlüter et al. 2012). More recently, emerging interdisciplinary approaches such as resilience theory, ecological economics, and complex adaptive system theory have contributed to an integrative study of SES, but these have often been criticized for their "ad hoc" approach to representing relevant variables and processes, particularly with respect to modeling changes in human behavior (Feola and Binder 2010).

There are many ways to describe and model complex SES so there is always the possibility that another representation might give a different outcome (Polhill and Gotts 2009). SES models are also often based on assumptions about human behavior or ecological dynamics that are uncertain but potentially have a substantial effect on model outcomes. Sources of uncertainty include, for instance, lack of knowledge on the decision making process of human agents, on how future benefits are valued, on how actors learn, or on the processes that are considered relevant for the dynamics of a natural resource, such as the reproduction and

${ }^{1}$ Department of Biology and Ecology of Fishes, Leibniz-Institute of Freshwater Ecology and Inland Fisheries, Berlin, Germany, ${ }^{2}$ Stockholm Resilience Centre, Stockholm University, Stockholm, Sweden, ${ }^{3}$ Global Climate Forum (GCF), Berlin, Germany, ${ }^{4}$ Technical University Delft, The Netherlands, ${ }^{5}$ Chair of Integrative Fisheries Management, Faculty of Agriculture and Horticulture, Humboldt-Universität zu Berlin, Germany 
growth of a natural resource. Modeling assumptions about these processes can be based on theories or empirical observations. Making explicit the assumptions a model is based on is thus of particular relevance to understand and evaluate model outcomes. This is particularly important in an interdisciplinary context where theories and assumptions that stem from different disciplinary backgrounds are often not known to researchers from other disciplines.

Several protocols have recently been proposed as a response to the difficulty of describing SES models in a way that their structure and processes are transparent and they can be assessed and reimplemented. These include the ODD and ODD+D protocols (Overview, Design concepts, and Details; Grimm et al. 2006, Müller et al. 2013) or the use of Web Ontology Language (Polhill and Gotts 2009). Others have suggested protocols, e.g., TRACE (Schmolke et al. 2010) or steps that define best practice for model development and evaluation (Jakeman et al. 2006) to improve modeling practice and disclose the modeling process itself to scrutiny. All of these protocols and best practices, however, are primarily about the process of model building, and less about the process of model conceptualization through abstraction from the target system.

The various challenges associated with model conceptualization outlined above, such as the integration of knowledge and theories from different disciplines, the variety of possible explanations scattered across disciplines, the uncertainties of social and ecological processes, and the sensitivity of model outcomes to the underlying assumptions, are partially due to the lack of a common analytical framework (Anderies et al. 2006, Jeffrey and McIntosh 2006). Recently, Ostrom (2007, 2009) proposed a general framework for SES that aims to organize variables involved in different theories and models of SES and that is commonly understood across disciplines. It provides a common set of variables for studying a single SES or for comparing similar SES. It is one of the most comprehensive frameworks available, and as such is particularly suited to address the above challenges. Furthermore, it is theory-neutral to the extent that it can be applied using a variety of approaches and theories and hence supports the development of different types of models. Given the interdisciplinary nature of SES research and the diversity of approaches to study their dynamics at different levels of aggregation, this is an important aspect.

Our aim is to assess how the SES framework (SESF; McGinnis and Ostrom 2014) in a formalized and refined version proposed by Hinkel et al. (2014) can help to develop and communicate models to analyze the dynamics of SES. In our application of SESF for modeling, we are particularly interested in two aspects. First, we want to test whether it can support model conceptualization by guiding a structured and rigorous process of selecting variables and integrating different theories relevant for the research question under investigation. Second, we want to investigate whether the proposed procedure can help make explicit and transparent the decisions and assumptions made during abstraction and model design. We briefly introduce SESF (McGinnis and Ostrom 2014) and the formalization and refinement proposed by Hinkel et al. (2014). We discuss how SESF can guide a model development process, particularly the first step of abstraction from the target system. We apply the proposed procedure to develop a model of fish stocking decision making in recreational fisheries as a case example. Recreational fisheries are a well-studied SES (e.g., Carpenter and Brock 2004, Biggs et al. 2009, Horan et al. 2011) in which social-ecological interactions between natural resources (fish), harvesters, and their institutions are strong and intimately coupled. We discuss how SESF facilitated the operationalization of a research question, and made the selection of key variables and relationships for modeling the SES easier and more efficient and transparent.

\section{ANALYZING PROCESSES AND OUTCOMES IN SES WITH THE HELP OF SEMANTIC AND PROCESS RELATIONSHIPS}

A framework can be defined as a set of core concepts that is likely to be involved in theories and models (Ostrom 2005). Within a particular framework, alternative theories are used to make broad predictions about the outcomes resulting from changes in variables (Ostrom 1999). Models implement theories using different formal techniques (Ostrom 2005). For our analysis of the dynamics of SES we make use of several types of semantic relationships between variables of SESF proposed by Hinkel et al. (2014) as well as process relationships that define dynamic interactions (see Appendix 1 for a representation of the SESF including semantic and process relationships in the Unified Modeling Language [UML]). This helps to systematically organize the many variables that are relevant for explaining outcomes in SES.

The introduction of process relationships is a further development of SESF that is particularly helpful to study the dynamics of SES. Process relationships formalize interactions between variables, e.g., the influence of an actor on the resource unit. The development of process relationships makes explicit which variables influence each other to determine the change of the coupled system over time. Process relationships thus provide a structured and transparent way to develop and communicate the structure of a model at a generic level. At this level we do not specify the relation itself, but only its type, which is the set of all possible relations. Specifying one particular relation would mean relating the variables through a specific statistical or mechanistic model, whereas SESF should remain theory-neutral, allowing for different models of the same process relationship. SESF therefore specifies a process relationship merely by naming the variables that participate in it. This suffices for making explicit the modeling decisions that we consider most crucial: which variables to include at what level of detail.

Process relationships are represented as mathematical (directed) relation types, with the influencing variables being the domain of the relation type, and the influenced variables its codomain. To represent that variable $\mathrm{A}$ and $\mathrm{B}$ influence variable $\mathrm{C}$ we write:

$$
(\mathrm{A}, \mathrm{B}) \rightarrow \mathrm{C}
$$

For example, the highest level process of a social-ecological system that consists of a governance system (GS), a resource system (RS), and an exogenous environment (ENV) can be represented as:

$$
(\mathrm{GS}, \mathrm{RS}, \mathrm{ENV}) \rightarrow(\mathrm{GS}, \mathrm{RS})
$$

This means that the future state at time $t+1$ of a SES comprising a governance system and a resource system is determined by the 
interactions of the governance system, the resource system, and the external environment at time t. This is the highest level of many more detailed reformulations that specify the variables that interact to produce the outcomes of interest. The relation type as represented in Equation 2 indicates which variables in the domain, i.e., the independent variables on the left-hand side, influence the variables in the codomain, i.e., the dependent variables on the right-hand side. Note that the environment (ENV) does not appear in the codomain because it is considered as exogenous and thus not influenced by processes within the system of interest.

We define outcome indicators through indicator relationships that combine the variables that have to be measured to answer a specific research question. They are particularly important because they determine which aspects of the system the analysis will focus on. Note that a model is always a specific representation of a system to answer a research question. Outcome indicators can be normative, e.g., an indicator that defines sustainability, or non-normative, e.g., an indicator that defines the ecological condition of a fish population. In a fishery context one might, for example, define an ecological indicator such as the spawning potential ratio (SPR; Mace 1994) to assess the ecological condition of an exploited fish population. In SESF this indicator is represented through a relation type that defines which variables determine the indicator:

\section{SpawningPotentialRatio \\ $\leftarrow$ (FishPopulation.NumberOfEggsOfFishedPopulation, \\ FishPopulation.NumberOfEggsOfUnfishedPopulation)}

The notation concept.variable, e.g. FishPopulation.NumberOfEggs, indicates that the variable after the period is attributed to the concept named before the period, e.g., the fish population has a number of eggs under unfished and fished conditions.

Finally, aggregation relationships define how variables that are measured at different levels or scales are aggregated. For example

$$
\text { NumberOfFish: Number[Ind.] } \leftarrow \text { Fish* }
$$

The asterisk indicates that many individual fish are aggregated to the variable NumberOfFish. Note that to distinguish them from process relationships, indicator relationships and aggregation relationships are denoted as (codomain) $\leftarrow$ (domain), and not (domain) $\rightarrow$ (codomain), and that their codomain typically comprises a single variable (the indicator or aggregate).

\section{FRAMEWORK-BASED DEVELOPMENT OF A SOCIAL- ECOLOGICAL SYSTEMS MODEL}

If we view a model as a representation of a system meant to conveniently, e.g., quickly, safely, cheaply, answer a specific question about that system (Rothenberg 1989), then different questions will lead to different models. As with any empirical investigation, the challenges in developing a SES model lie in making the research question operational, particularly in the selection of variables and relationships that are relevant to explain observed or hypothesized dynamics. When selecting the variables of interest for our specific analysis we also need to determine which variables change over the time scales considered (state variables), which stay constant (parameters), what structural elements drive or constrain interactions or processes, and how they interact with other variables in the system over time. The result of the process of abstraction from a given target system is a conceptual model or case-specific framework that serves as the basis for data collection, design of the formal model, and its implementation and analysis. It is this first step of system analysis and conceptual model development where we see the main contribution of SESF. It is also the phase where simplifications and assumptions have to be made that can potentially have significant effects on model outcomes. Hare and Pahl-Wostl (2001) for instance show that the way learning of actors is represented can strongly affect the outcomes of a model.

SESF can support the process of abstraction of the target system with respect to a given research question in several ways (Fig. 1). In a forward-reasoning approach (Fig. 1, left-hand side), SESF can help modelers and domain experts to identify variables that are considered relevant to answer a given research question. This provides for a more systematic and comprehensive selection of relevant variables. The selected variables are then the fundamental building parts for processes and indicators. When defining processes and indicators, the often normative decisions on which the relation types are based can easily be made explicit by motivating why certain variables are included while others are not.

Fig. 1. The framework-supported modeling process with a forward and backward reasoning process for the identification of relevant variables and relationships. SES = social-ecological systems.

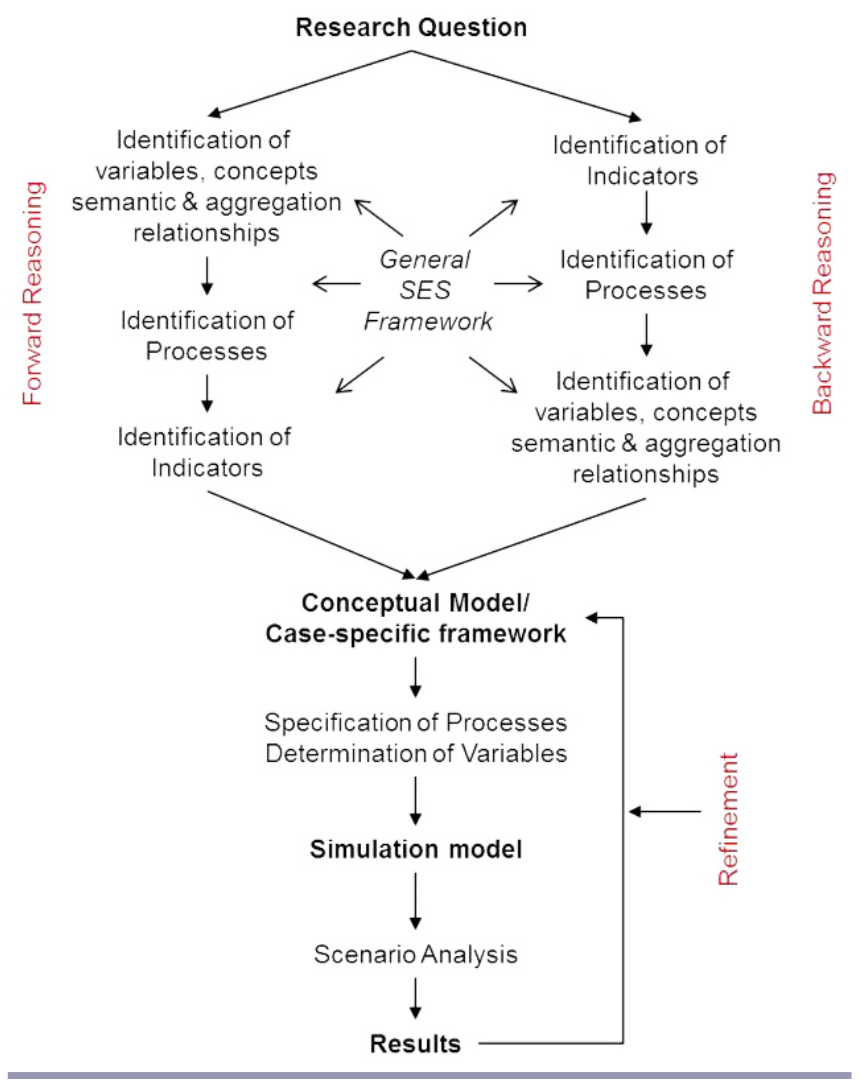

In a backward-reasoning approach (Fig. 1, right-hand side) the identification of relevant variables and processes is directly driven by the research question, i.e., the goal of the investigation. The 
operationalization of the research question yields the indicators used to measure the outcomes. The variables in the indicator relationships that are not static are determined by processes, which consequently have to be identified next. Again this procedure can support making explicit the choices made when determining indicator and process relationships. Variables that become relevant when developing the processes and indicators are then added to the case-specific framework, and their semantic and aggregation relationships are specified. SESF can help with the identification of variables that are potentially relevant for the target system and question of interest and need to be included in process relationships. It can also assist in clarifying concepts that are used with different meanings or different names across disciplines, such as "flow" versus "transaction" in ecology and economics, respectively.

In practice, a combination of a forward and backward-reasoning approach is likely to be most helpful. The case-specific framework that results from this abstraction process serves as the basis for the design of the formal model. The next steps are the specification of processes, i.e., choosing specific mathematical or algorithmic representations of causal relationships, and the determination of values of variables. The values of parameters and the initial values of state variables have to be defined based on empirical data or expert assessment. The model can then be studied using mathematical analysis or computer simulations to answer the research question. The results will also help to refine the framework and provide a test for assumptions about causal relationships that drive the dynamics of the system.

\section{APPLICATION OF THE FRAMEWORK FOR MODEL- BASED ANALYSIS OF THE COEVOLUTION OF A RECREATIONAL FISHERY}

Recreational fisheries across much of central Europe are organized in local angling clubs that have the right to fish but also the duty to manage their water bodies sustainably and in line with fisheries and nature conservation legislation (Daedlow et al. 2011). In this context, stocking, defined here as the deliberate release of fish originating from other water bodies or hatcheries into a particular water body, is a common management practice to maintain or enhance fish stocks and angler well-being (Cowx 1994). The ecological impacts and socioeconomic benefits of stocking are uncertain (Lorenzen et al. 2010), as is the response of anglers to these measures (Rogers et al. 2010). Ecological uncertainty arises from environmental variability, e.g., interannual water temperature fluctuations that affect fish population growth or from competition and predation between the stocked and wild fish or other species of the community. Social uncertainty arises from the fact that the responses of anglers to stocking measures, such as a change in future angling behavior that results from changes in the satisfaction anglers derive from their angling experience, are usually only poorly known (Fulton et al. 2011, van Poorten et al. 2011).

Modeling can be a helpful tool to analyze how the most salient social-ecological interactions in a fishery with stocking play out over time and determine its sustainability. If, for instance, anglers demand for more stocking because of angling norms and dissatisfaction with their current catch rate, and the club manager decides to respond by stocking, the dynamics of the fish population will change affecting future catch and anglers' responses. It is these feedbacks that can lead to unexpected or undesired developments of the SES with implications for its sustainability (van Poorten et al. 2011). A model is a helpful tool to analyze these feedbacks under different assumptions about adaptive responses of club managers and choices of stocking measures to enhance understanding of the resulting dynamics and potential outcomes.

A stocking decision of a club manager involves a choice of the number, the size, and origin of fish of a particular fish species to be stocked. The success of a stocking measure is influenced by the size of the stocked fish, the conditions in the water body, and the number of fish in the stocked and wild fish population because all of these factors impact the competition between wild and stocked fish (Lorenzen 2005). Practical stocking guidelines in Germany often recommend stocking small-sized fish because it is believed that they can more easily adapt to the conditions in the water body and the problem of overstocking is supposed to be avoided because of natural regulation (Baer et al. 2007). However, there are alternative perspectives that larger sized fish might offer better returns because of higher survival in the stocked water system, but this comes at a higher cost of production (Lorenzen 2005). We develop a dynamic simulation model to test the possible implication of deviating from the practical recommendation of stocking only small-sized fish into a wild stock in a locally managed SES with tight coupling between the ecological, the angler, and the manager system, similar to van Poorten et al. (2011). The aim of the model is to answer the following research question: How does the size of stocked fish influence the sustainability of a locally managed recreational fishery when the stocking decision is driven by the catch satisfaction of bounded rational anglers?

We illustrate the application of SESF for the development of a model to answer this question. Note that the example only focuses on selected aspects of the model for the sake of readability and space. It is intended to illustrate the use of the framework, not to provide a complete description of the modeling process and the model.

Following SESF, a recreational fishery SES at the highest tier consists of a governance system, actors, some of which are anglers, as a subconcept of actors; and waterbodies, as a subconcept of resource systems, which can have one or more exploited and unexploited fish populations. The target fish population is an aggregation of individual fish (resource units) and thus a subconcept of the population of resource units. Note that the distinction between different levels of aggregation such as resource unit and population of resource units in the SES framework has been introduced by Hinkel et al. (2014) because McGinnis and Ostrom (2014) do not allow making this distinction. These first tier concepts have various variables attributed to them. We follow a backward reasoning approach guided by the research question and SESF to identify those attributes that are relevant to explain stocking outcomes and hence should be included in the model. This procedure leads to the case-specific framework or conceptual model that forms the basis of a formal model of a stock-enhanced recreational fishery. Variables that are directly taken from SESF are written in italics. 


\section{Identification of outcome indicators}

As indicated by the research question we are interested to understand how the size of stocked fish influences the sustainability of a locally managed fishery. The first step in operationalizing the research question is thus to define "size of stocked fish" as the control variable, and "sustainability of a locally managed recreational fishery" as the outcome indicator. We operationalize "sustainability" with several indicators that relate to the ecological and social dimensions of the recreational fishery SES (social and ecological performance measures). Ecological sustainability is defined as the rate at which wild-origin fish are replaced by hatchery-origin fish, i.e., the replacement rate. Replacement of wild fish by stocked fish can affect the genetic diversity of the fish population, which might negatively influence its capacity to adapt to changing future conditions (Laikre et al. 2010). Social sustainability is defined as the welfare of all the anglers in the angling club (angling club welfare) as represented by user satisfaction or utility (Arlinghaus 2006). We formalize the outcome indicators in the form of function types. For readability, we abbreviate GovernanceSystem to GS, AnglingClub to AC, AnglerGroup to AG, FishPopulation to FP, and Environment to ENV.

\section{Ecological outcome indicator}

Replacement rate (replacement of wild-origin fish by hatcheryorigin fish)

ReplacementRate

$\leftarrow$ (FP.NumberOfFish, FP.NumberOfHatcheryOriginFish)

Social outcome indicator

Angling club welfare

$$
\text { AnglingClubWelfare } \leftarrow \text { (AG.Satisfaction) }
$$

The variables NumberOfFish and NumberOfHatcheryOriginFish that determine the replacement rate are subconcepts of the second-tier variable PopulationOfResourceUnits. NumberOfUnits in SESF. Satisfaction is not a variable in SESF. We introduce it here as a new variable of the actor group "angler group" because having satisfied users is a primary goal of fisheries' management in angling clubs (Royce 1983), i.e., if a large number of anglers are satisfied with their angling experiences it is assumed that the well-being of the club is high.

\section{Specifying the process relationships}

The second step in operationalizing the research question is specifying the variables that codetermine changes in the state of another variable and organize them in process relationships. Relevant are those processes that explain changes in state variables that are in the domain of the outcome indicator function types given above. Processes can be developed top-down by starting at the highest level of aggregation and then progressively unpacking the system until changes in all relevant variables are explained. Note that for each process relationship a narrative should be developed first, which helps to identify the variables of the domain.

In our example, the variables in the domain of the indicator function type that we need to further explain are: the number of fish in the fish population and angler satisfaction. The change in the number of fish over time is influenced by attributes of the fish population itself, e.g., degree of density-dependent population regulation (Lorenzen 2005), as well as those of the angling club, e.g., number of stocked fish, the group of anglers, and the environment (related ecosystem), which at the highest level can be expressed by the following relation type:

$$
\text { FPGrowth: (FP, AC, AG, ENV) } \rightarrow \text { FP.NumberOfFish }
$$

By further unpacking the lower-tier variables of $\mathrm{FP}, \mathrm{AC}$, and $\mathrm{AG}$, we can specify that the number of fish in the next time step is influenced by the current number of fish, the growth rate of the fish population, the productivity of the water body, the exploitation by anglers (catch), the amount of fish stocked into the population by the angling club, as well as some external climatic influences as expressed in this relation type:

FPGrowth: (FP.NumberOfFish, FP.GrowthRate, WaterBody.Productivity, AG.Catch, AC.NumberOfStockedFish, AC.SizeOfStockedFish, ENV) $\rightarrow$ FP.NumberOfFish

Although the growth rate and productivity of the system are variables of SESF, the variable catch (or harvesting level) is currently not represented. It is an outcome from the process of angling, i.e., the interaction of the anglers with the fish population. We attribute catch to the concept angler group because it affects the future actions of anglers. We assume that the catch of the angler group is influenced by the total effort of the anglers, i.e., the hours they spend angling, the angling gear used (Actor.TechnologiesAvailable), and the current number of fish in the fish population. We introduce effort as another new variable in SESF attributed to the AnglerGroup because of its key importance in determining the number of fish that are extracted from the fish population.

$$
\begin{aligned}
& \text { Angling: (AG.Effort, AG.AnglingGear, FP.NumberOfFish) } \\
& \rightarrow \text { AG.Catch }
\end{aligned}
$$

The catch an angler obtains is also an important determinant of the satisfaction he derives from an angling experience. We need to measure angler satisfaction to determine the social outcome indicator. Empirical evidence has shown that angler satisfaction is strongly influenced by the relation between catch expectations and the actually realized catch (Arlinghaus 2006). In this model, we thus assume that past and present catch rates are the dominant, and for simplicity the only, variables that influence the satisfaction of anglers. Changes in the satisfaction of anglers are formalized in the process EvaluateBenefits as follows:

$$
\begin{aligned}
& \text { EvaluateBenefits: (AG.Catch, AG.CatchExpectation) } \rightarrow \\
& \text { AG.Satisfaction }
\end{aligned}
$$

The relation type of the process EvaluateBenefits makes explicit our assumptions about the variables that explain the satisfaction of the angler group. These assumptions vary depending on the chosen theoretical perspective or empirical evidence. For example, angler satisfaction might not exclusively depend on the number of fish in the catch, but also on the experience of crowding and some other attributes of the catch, such as the size of the fish caught (Hunt 2005). In this case the relation type would be formalized as follows.

EvaluateBenefits2: (AG.Catch.Number, AG.Catch.MaxSize, AC.Crowding, AG.CatchExpectation) $\rightarrow$ AG.Satisfaction 
Fig. 2. Concepts, variables, and semantic relationships of the recreational fishery that are included in the model to assess the impact of the size of stocked fish on the sustainability of the fishery. Grey boxes indicate the specification of the general framework for the recreational fishery's social-ecological systems (SES). Downward arrows represent relationships in which a variable is attributed to a concept, e.g., the recreational fishery has several actor groups. The upward arrows with open arrow represent relationships in which subconcepts are a type of the more general concept, e.g., an angler group is an actor group.

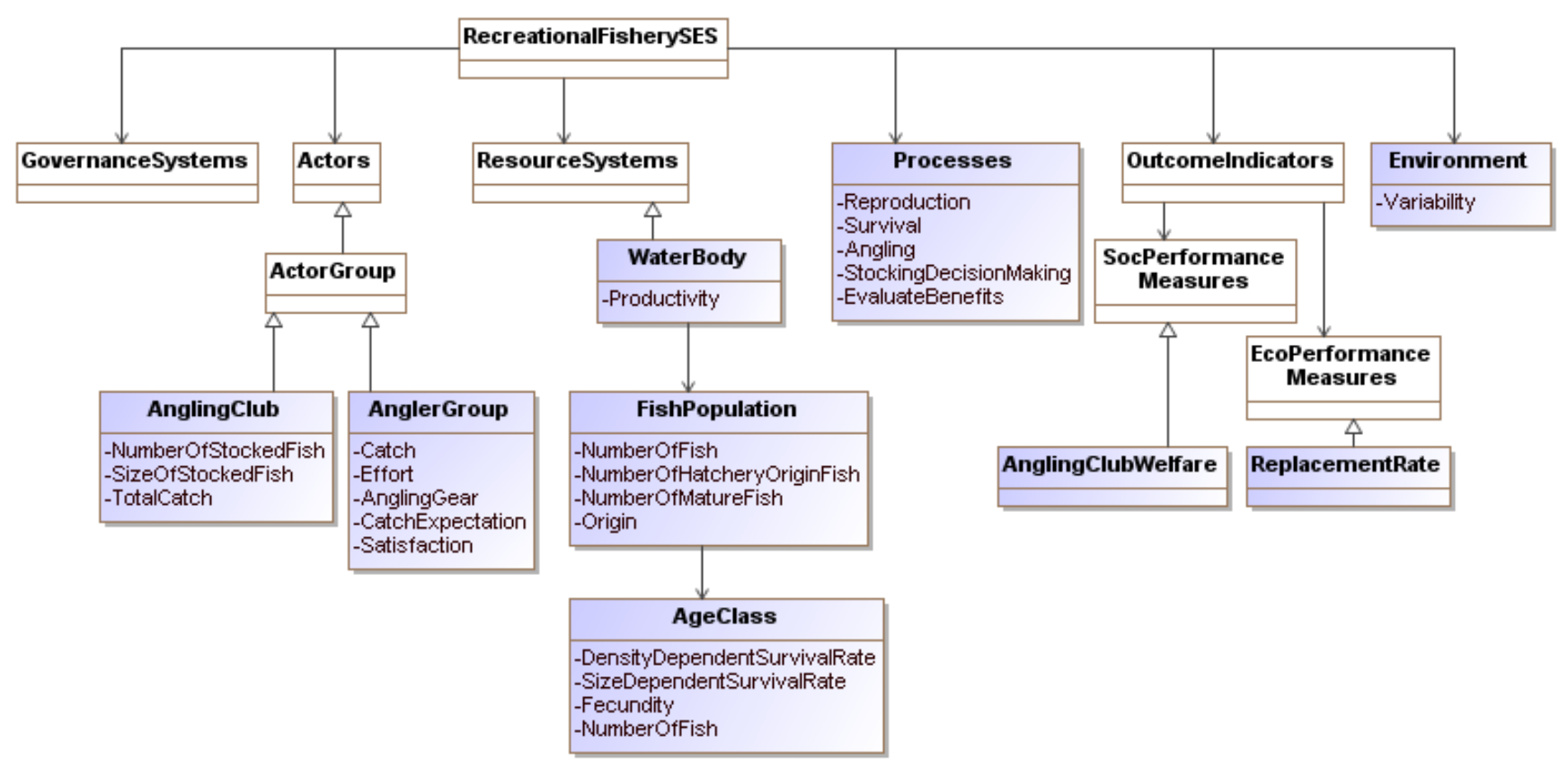

Note also that the relation type in Equation 9a does not determine how exactly the catch and catch expectations influence angler satisfaction. We specify the assumed causal relationships when we model the processes in the next step.

The growth of the fish population also depends on the number of stocked fish, which is determined through the process of stocking decision making. We model this process explicitly because we want to understand the sustainability of a fishery where stocking decisions are adaptive, based on the satisfaction of anglers. There is empirical evidence that stocking decision making in angling clubs is strongly influenced by anglers' satisfaction because of anglers' perceptions and norms about fishery management (van Poorten et al. 2011). Anglers react to these norms by pressuring angling club managers to stock. We follow van Poorten et al. (2011) and assume that changes in angler satisfaction directly determine the amount of fish stocked by the angling club each year, which may go up or down incrementally, and that no other alternative actions are considered by the manager (Deadlow et al. 2011).

StockingDecisionMaking:

(AC.NumberOfStockedFish, AG.Satisfaction) $\rightarrow$

AC.NumberOfStockedFish

\section{Determining the level of aggregation}

In the example above we successively unpacked the processes and variables needed to answer our research question in a transparent and rigorous way. This procedure also involves choices about the level of aggregation at which interactions or processes should be represented. There is need to decide at which level, e.g., at the level of individuals or populations, we want to relate variability and heterogeneity of the dependent, e.g., amount of stocking, and independent, e.g., angler satisfaction, variables. In some cases, for example, variability at the individual level, of an individual fish or social actor, will be crucial in determining outcomes (Johnston et al. 2010), whereas in other cases more aggregate processes play out. The hierarchy of concepts and variables provided by SESF can help to explain the choices of the level and method of aggregation. Both should be justified by underlying key processes such as age or size-based transitions in the life cycle, variation in attributes of individual fish, such as their size that affect angler utility and choices, or the importance of fish size for stocking outcomes.

For instance, we have to be able to identify whether a fish in the fish population is a wild fish or originated from a hatchery to account for size-dependent competition between wild and stocked fish (Lorenzen 2000) and measure the replacement rate of wild fish. The fish population growth process can thus not be modeled at the level of a single population. However, it does not need to be modeled at the individual level either, as long as we assume that the fish only differ with respect to characteristics that are related to their origin, such as their survival rates. In this case we can model hatchery-origin and wild-origin fish at the level of two subpopulations of fish similar to Lorenzen (2005) and Rogers et al. (2010). Consequently, we introduce origin (hatchery or wild) as a new variable of the FishPopulation. We also need to further 
unpack the FPGrowth process by further decomposing what is now represented as a growth rate into fish population survival and reproduction at different sizes. We do so by modeling survival and reproduction disaggregated into different age classes (as a proxy for different sizes) so that we can account for different survival rates of different sized fish (van Poorten et al. 2011).

\section{The conceptual model}

The development of indicator and process relationships as described above has helped to identify concepts and variables and the level of aggregation needed to analyze the dynamics of the recreational fishery SES with respect to our research question (Fig. 2). The development of the processes and the hierarchy of concepts and variables proceeds in an iterative way, where the development of processes helps to refine the variables, and the development of the conceptual relationships between variables helps in the development of processes.

The variables in the domain and codomain of process relationships can also be graphed in form of an influence diagram. Influence diagrams are helpful to highlight the main feedbacks that are driving the coevolution of the stock-enhanced recreational fishery system (Fig. 3). On the right-hand side, angler satisfaction influences the number of stocked fish, which together with the survival and reproduction (represented as two circles to the left of the variable NumberOfFish) and angling influence the number of fish in the fish population. The catch anglers receive from the fish population and their catch expectation that formed from past experiences in turn affects their satisfaction, which drives stocking in the next year. It is important to note that influence diagrams contain less information than the set of process relationships derived above. Most critically, influence diagrams do not display the names of the processes or the information on how various variables coinfluence each other. Influence relationships only relate two variables, whereas process relationships relate all variables that inseparably codetermine changes in the influenced variables. Understanding processes in the sense of these codeterminations is a necessary step in understanding the dynamics of SES that influence diagrams cannot provide.

\section{Modeling of processes}

The next step in operationalizing the research question is the mathematical modeling of the process relationships that have been specified above. Mathematical or rule-based functions are to be defined that determine how given values for the variables in the domain of the process relationship lead to values for the variables in the codomain. Mathematically speaking, the functions must be of the type given by the process relation. The modeling of process relationships makes explicit the theoretical or empirical assumptions about causal relationships between the variables in the domain. In our recreational fisheries model for instance, ecological theories of population dynamics are used to model the survival and recruitment of the fish population. The representation of social dynamics is based on empirical observations about angler behavior and decision making in angling clubs (summarized in van Poorten et al. 2011) as well as the theory of reinforcement learning (Sutton and Barto 1998) and bounded rationality (Simon 1957).
Fig. 3. Influence diagram based on the relations defined for the recreational fishery example. The two feedback cycles to the left of the variable NumberOfFish represent the reproduction and survival processes.

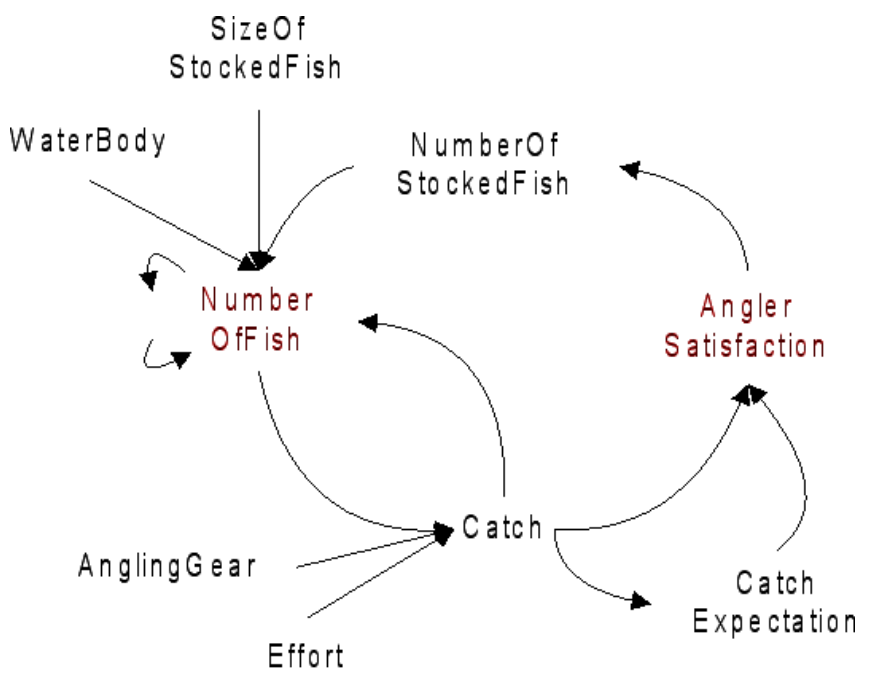

We illustrate the modeling of processes by the examples of the population growth of the wild subpopulation and stocking decision making. We have argued for the need to model the fish population disaggregated into age classes to be able to take different survival rates of different sizes of fish into account. Agestructured models are standard approaches in fisheries to assess changes in the age-structure of exploited fisheries (e.g., Hilborn and Walters 1992). The fish population growth process consists of the processes of fish reproduction and fish survival (e.g., Ricker 1954, Beverton and Holt 1957, Wootton 1998). They are determined by the number of mature fish in the population as well as the survival rates, fecundity, and vulnerability to fishing of each fish of a specific size or age class.

Reproduction: (FP.NumberOfMatureFish, FP.AgeClass.Fecundity,
FP.AgeClass.DensityDependentSurvivalRate, ENV) $\rightarrow$ FP.AgeClass.NumberOfFish

Survival: (FP.AgeClass.NumberOfFish,

FP.AgeClassDensityDependentSurvivalRate,

FP.AgeClass.SizeDependentSurvivalRate, AC.TotalCatch) $\rightarrow$

FP.AgeClass.NumberOfFish

Based on the literature we assume that the recruitment into the first age class, i.e., fish from age 0 to age 1 , is strongly densitydependent (Myers and Cadigan 1993) whereas the survival of larger, so-called recruited fish is primarily size-dependent (Lorenzen and Enberg 2002). These assumptions are included in the formulation of the specific functions to represent recruitment and age specific survival that we adapted from Lorenzen (2005). 
Reproduction:

$$
N_{W, 1, t+1}=s_{1} * f * S_{w, t}
$$

Survival:

$$
\begin{aligned}
& N_{W, a+1, t+1}=N_{W, a, t}- \\
& \left(\left(\frac{L_{a-1, t}}{L_{a-1, t}+L_{\infty}(B) *\left(e^{K}-1\right)}\right)^{\frac{M_{1}}{L_{\infty}(B) * K}}+q * E * V_{a}\right) * N_{W, a, t} \\
& a=\{1,2, \ldots \text { agemax }\}
\end{aligned}
$$

Where $N_{W}$ is the wild fish component, $a$ is the age class; $s_{1}$ the density-dependent survival of juveniles, $f$ the relative fecundity, $S_{W}$ the number of mature fish in the wild fish population, $L_{a}$ the length of fish in age class $a, L_{\infty}(B)$ the maximum length at biomass level $B, M_{1}$ the natural mortality rate at unit length (Lorenzen $2000), K$ the competition coefficient, $q$ the catchability, $E$ the total angling effort, and $V_{a}$ the vulnerability of fish in age class $a$ to angling.

The process StockingDecisionMaking consists of the variables satisfaction of anglers and the number of stocked fish (Equation 10). In the absence of knowledge of how angler satisfaction functionally affects stocking decision making, we follow van Poorten et al. (2011) and assume a very simple linear relationship where the current stocking density is increased or decreased proportionally to the angler satisfaction, thus simulating a manager that immediately responds to changes in angler satisfaction.

StockingDecisionMaking:

(AC.NumberOfStockedFish, AG.Satisfaction) $\rightarrow$ AC.NumberOfStockedFish

$$
D_{t+1}=D_{t}+d * U_{t}
$$

where $D_{t}$ is the amount of fish stocked at time $t, U_{t}$ the angler satisfaction at time $t$, and $d$ a scaling factor. The implication of this functional choice and the choice of the intensity of the coupling between number of stocked fish and angler satisfaction is assessed during model analysis through testing alternative values for the parameter $d$ and different functional relationships for the number of stocked fish.

The modeling of processes is informed by the theory or approach that frames the research question. In our case this is the assumption that recreational fisheries codevelop through adaptive behavior of actors and resulting ecological dynamics that influence each other through feedback relationships. If the research question is based on a different theory, such as control theory where it is assumed that the manager maximizes some index of social welfare over a specified time horizon and a given discount rate, the StockingDecisionMaking process would be specified using a different function. The amount of stocking would in that case be determined by maximizing catch or angler well-being as a function of a stock-enhanced fish population, where stocking is costly.
Finally, once the mathematical models are specified, they can be analyzed analytically or implemented in computer code for simulations. Although this step is not a trivial one it goes beyond the scope of this paper. Note that when implementing the specified processes as a computer program, their sequence becomes important. Likewise the handling of initial conditions can be challenging, particularly in cases with history and memory as in our process relationship "EvaluateBenefits" (Equation 9a). Once implemented, the simulation model can then be used for scenario analysis to answer the research question: How does the size of stocked fish influence the sustainability of a locally managed recreational fishery when the stocking decision is driven by the catch satisfaction of bounded rational anglers? This means that the control variable, in our case the size of stocked fish, is varied and the resulting social-ecological dynamics, management responses (stocking), and long-term impact on the defined sustainability outcomes (replacement of wild fish stock, and social welfare) is assessed. In the Appendix 2 we show some modeling results that indicate that replacement of wild fish is highest when stocking juvenile fish of medium size. In this case wild fish are replaced when the memory of anglers of past angling experiences is medium or high (Figure A2.2b). When stocking larvae there is no replacement of wild fish (Figure A2.2a) despite high stocking densities (Figure A2.1a) because of the low chance of survival of stocked larvae. Hence local management that is based solely on angler satisfaction can lead to increases in yield (Figure A2.1b) but replacement of wild fish.

\section{DISCUSSION}

Understanding the dynamics of SESs requires identifying and analyzing the various social-ecological processes that determine the outcomes of interest. We have developed a structured stepwise procedure based on SESF, indicator and process relationships to identify these processes and conceptualize a simulation model to analyze their interactions. First, a backward reasoning approach is used to identify the relevant variables and processes by systematically unpacking the system to the level of detail needed to answer a specific research question. SESF guides this process through providing a set of commonly used variables and a set of semantic and process relationships that help conceptualize the structure and interactions of the SES in a consistent and transparent way. The result of the first phase of the procedure is a conceptual model or case-specific framework that specifies the variables and relationships of the SES that determine the outcomes of interest. In the second phase, the selected processes are modeled, i.e., cause-effect relationships between variables are defined based on theories or empirical observations. This results in a dynamic model that can subsequently be analyzed mathematically or through simulations. Throughout the whole procedure of model conceptualization and process modeling the analyst is encouraged to specify and explain why a certain variable or process is included, and based on which theory or empirical evidence, thus enhancing the transparency of the modeling process.

SESF is at the core of this procedure because it provides a set of general concepts, variables, and relationships that have proven to be relevant in explaining outcomes in a large number of SES. The generality of SESF and the fact that processes are represented as relation types allows one to link variables in any way reflecting different theories or empirically-based assumptions of the dynamics of the SES. This allows for a combination and 
comparison of different approaches and theories to study a given SES and therefore achieve a more multifaceted insight into their dynamics. In the example given above we use coevolutionary theory (Norgaard 1994, Jeffrey and McIntosh 2006, Rammel et al. 2007) to develop a model that explores the consequences of adaptive behavior of decision makers that stock fish populations as a response to changes in angler satisfaction. The model provides insights on possible outcomes of local decision making when stocking decisions are based solely on angler satisfaction. Depending on the size of stocked fish and the memory of anglers of past catch experiences there can be little or very severe replacement of wild fish with variable effects on angling club welfare. Likewise SESF can be used to develop a model based on optimization theory to identify optimal stocking strategies given budgetary constraints, or an agent-based model to investigate the implications of diverse angler preferences on stocking outcomes. The developed process relationships will reveal that the selected variables and the level of aggregation at which processes are represented vary significantly between these different approaches.

The added value of using SESF for model development lies in the guidance it provides for (a) a comprehensive search for relevant variables from both the ecological and social domains, and (b) a systematic and transparent process of abstraction. The former is crucial for dealing with the complexity of SES and the multitude of potentially relevant variables and interactions, and for achieving an integrative analysis that addresses the coupled nature of SES. A systematic process of abstraction is valuable in that it ensures that no relevant variables are missed. The iterative process of developing a concept hierarchy and the process relationships functions as a cross-check that guides a systematic unpacking of all variables needed to explain the outcomes. For example, when developing a process relationship a variable may become relevant that was not included in the concept hierarchy before, such as, angler satisfaction or effort in our example. Conducting an integrative analysis is often challenging because of the bias of a model developer toward the system view with which she is most familiar. An ecologist for instance is likely to put more detail into the representation of the ecological dynamics while an economist is likely to stick to a rather simple representation of the ecological processes that produce the resource of interest (Schlüter et al. 2012). Both biases can lead to an incomplete or unrealistic representation of the dynamics of the coupled SES because of the neglect of important social or ecological processes.

The SESF-based procedure of model conceptualization also encourages the analyst to make explicit the reasons why she decided to include a variable or process at an aggregate level, such as the level of a population or community, or disaggregate to the level of individual interactions. The selection of the level of detail is a very important issue when analyzing complex SES because of the potential relevance of micro-scale variability for explaining macro-scale outcomes (see e.g., Smith and Wilen 2003). It is determined by the research question and our understanding or hypotheses of the relevant heterogeneity in a system. In our example of the fishery, knowing the number of fish in the fish population is not enough because we need to distinguish between hatchery and wild origin fish to calculate replacement. A systematic process of defining indicators and identifying variables following the above mentioned procedure can assist in making these choices.

By encouraging the modeler to make explicit modeling decisions and assumptions, the procedure enhances the transparency of the model. This is important for communication, model analysis, and interpretation of model results in interdisciplinary studies of SES, and for facilitating comparisons of models across different cases and theoretical foundations. Transparency is particularly relevant in an inter- or transdisciplinary context in which ontologies differ and underlying assumptions, e.g., on human decision making, are not common knowledge among participants from different disciplines or between modelers and model users. For instance in our example of stock-enhanced recreational fisheries the procedure makes explicit the assumption that anglers compare past with current experiences in forming their expectations of a good catch and do not consider possible future options. As such modeling decisions, particularly those related to the representation of human decision making and learning, can have significant impacts on model outcomes that need to be considered when analyzing and interpreting model results.

SESF allows any kind of explanation for an observed outcome, however, the choices made while specifying a concept hierarchy and process relationships constrain the explanations possible. Our testing of SESF for model development also shows that it can be difficult to stay true to the principle of going from higher levels of aggregation to lower ones, because our knowledge of some aspects of the SES might be more detailed than of others. When developing a model of a SES there is generally a temptation to go into too much detail. The framework-based procedure can help to avoid this by forcing the analyst to explain the reasoning for each additional variable when introducing it. There are, however, other crucial steps of model development or of an empirical investigation that SESF cannot support. One of them is the selection of system boundaries or of the focal level of analysis as well as the relevant spatial and temporal scales. Those decisions are dependent on the research question and the purpose of the model and need to be made before one starts to conceptualize the model. Another minimal aspect for the development of a SES model is the involvement of an interdisciplinary team with social and natural scientists. The proposed procedure is aimed to enhance communication between members of such an interdisciplinary team.

Aside from the example given in this paper there are several other possible applications of SESF in the field of SES modeling. It facilitates, for example, communication between modelers and experts or between modelers with different backgrounds (own observations). Another application is its use for model comparison for which it seems very suitable (see also Parker et al. 2008 for a framework to compare land-use and land-cover change models). SESF can help to find similarities and differences between models, e.g., whether different variables are included in a process relationship, whether authors added other functions to represent the same observation, etc. Furthermore, the concept hierarchy and processes developed for one study can be reused to develop other models. In doing so it will get more and more detailed. On the other hand if somebody wants to develop a simple model he can use only parts of it. 


\section{CONCLUSIONS}

In this paper we have shown how the SES framework combined with a structured approach that makes explicit conceptual and process relationships between variables as proposed by Hinkel et al. (2014) can support the process of developing a dynamic model of a SES to study social-ecological interactions and their outcomes over time. We have illustrated the proposed procedure of model conceptualization using SESF by applying it to an example from recreational fisheries. The exercise has shown that the procedure facilitates a more systematic and rigorous identification and definition of variables and process relationships that define the simulation model. The added value of using the proposed SESF-based procedure for a model-based analysis of the dynamics of a SES lies in its support of a systematic, integrative, and transparent process of model conceptualization. This enhances the communication of underlying model assumptions and encourages making explicit the theories and empirical observations that underlie modeling choices. This transparency is needed to allow for a critical assessment of model results, particularly in an interdisciplinary context. A critical reflection on the decisions about which variables and processes to include, at what level of aggregation, and based on which assumptions or theories can, in combination with a good model analysis, help assess the consistency of the model and the robustness of outcomes. This, in turn, allows for a quality check and an assessment of the value of a dynamic model for explaining the outcomes of interest.

The application of SESF for systematic model conceptualization complements protocols available for the description of simulation models (ODD; Grimm et al. 2006) and documentation of the model building and analysis process (TRACE; Schmolke et al. 2010) by providing guidelines and a format for documenting the choices made when abstracting from the target system to develop a conceptual model. This step is not explicitly treated in the other protocols. We suggest others test the usefulness of the SES framework-based approach when building SES models. We hope that by doing so we can start understanding general patterns, processes, and consequences that are particular across a range of SES and drive their dynamics and outcomes. Last, although SESF provides guidance and a procedure for the development of models of SES, these models can also inform the further development of the framework. Our application of the framework for the development of a SES model was motivated by the aim to study the dynamics of SES that lead to certain outcomes. The process relationships specified when conceptualizing the model are a first step toward including dynamics more explicitly into the framework. The highest level concept Activities and Processes could be extended with other generic processes in SES such as those relevant for the reproduction or replenishment of the resource, e.g., population growth, or for interactions between resource users and resources beyond harvesting such as recreation. Some elementary processes that can be found in many SES are currently under development for SESF; however, it remains an open question how specific we can get in representing process relationships in the core framework.

Responses to this article can be read online at: http://www.ecologyandsociety.org/issues/responses. $\mathrm{php} / 5782$

\section{Acknowledgments:}

We thank the members of the SESclub for many stimulating discussions that initiated and greatly informed the development of this manuscript. We would also like to thank the members of the Department of Resource Economics at Humboldt University, Berlin and the Department of Ecological Modeling at the UFZ-Helmholtz Centre for Environmental Research Leipzig-Halle for their critical comments and discussion of presentations of this work. We are particularly grateful to Thomas Falk and Ulrich Frey for their valuable feedback that greatly helped to improve an earlier version of the manuscript. Funding for MS and RA was provided by the German Ministry for Education and Research within the project Besatzfisch in the Program for Social-Ecological Research (Grant 01UU0907) and by the European Union through an ERC grant to $M S$.

\section{LITERATURE CITED}

Anderies, J., B. Walker, and A. Kinzig. 2006. Fifteen weddings and a funeral: case studies and resilience-based management. Ecology and Society 11(1): 21. [online] URL: http://www. ecologyandsociety.org/vol11/iss1/art21/

Arlinghaus, R. 2006. On the apparently striking disconnect between motivation and satisfaction in recreational fishing: the case of catch orientation of German anglers. North American Journal of Fisheries Management 26:592-605. http://dx.doi. org/10.1577/M04-220.1

Arlinghaus, R., and T. Mehner. 2005. Determinants of management preferences of recreational anglers in Germany: habitat management versus fish stocking. Limnologica 35:2-17. http://dx.doi.org/10.1016/j.limno.2004.10.001

Baer, J., K. Blasel, and M. Diekmann. 2007. Benefits of repeated stocking with adult, hatchery reared brown trout, Salmo trutta, to recreational fisheries? Fisheries Management and Ecology 14:51-59. http://dx.doi.org/10.1111/j.1365-2400.2006.00523.x

Baumgärtner, S., C. Becker, K. Frank, B. Müller, and M. Quaas. 2008. Relating the philosophy and practice of ecological economics: the role of concepts, models, and case studies in interand transdisciplinary sustainability research. Ecological Economics 67:384-393. http://dx.doi.org/10.1016/j.ecolecon.2008.07.018

Berkes, F., and C. Folke, editors. 1998. Linking social and ecological systems: management practices and social mechanisms for building resilience. Cambridge University Press, Cambridge, UK.

Beverton, R. J. H., and S. J. Holt. 1957. On the dynamics of exploited fish populations. Fishery Investigations Series II. Ministry of Agriculture, Fisheries and Food, London, UK.

Biggs, R., S. R. Carpenter, and W. A. Brock. 2009. Turning back from the brink: detecting an impending regime shift in time to avert it. Proceedings of the National Academy of Sciences 106:826-831. http://dx.doi.org/10.1073/pnas.0811729106

Carpenter, S. R., and W. A. Brock. 2004. Spatial complexity, resilience, and policy diversity: fishing on lake-rich landscapes. Ecology and Society 9(1): 8. [online] URL: http://www. ecologyandsociety.org/vo19/iss1/art8/ 
Carpenter, S. R., H. A. Mooney, J. Agard, D. Capistrano, R. S. DeFries, S. Díaz, T. Dietz, A. K. Duraiappah, A. Oteng-Yeboah, H. M. Pereira, C. Perrings, W. V. Reid, J. Sarukhan, R. J. Scholes, and A. Whyte. 2009. Science for managing ecosystem services: beyond the Millennium Ecosystem Assessment. Proceedings of the National Academy of Sciences 106:1305-1312. http://dx.doi. org/10.1073/pnas.0808772106

Cowx, I. G. 1994. Stocking strategies. Fisheries Management and Ecology 1:15-31. http://dx.doi.org/10.1111/j.1365-2400.1970. $\underline{\mathrm{tb} 00003 . \mathrm{x}}$

Daedlow, K., T. D Beard, Jr., and R. Arlinghaus. 2011. A property rights-based view on management of inland recreational fisheries: contrasting common and public fishing rights regimes in Germany and the United States. American Fisheries Society Symposium 75:13-38.

Feola, G., and C. R. Binder. 2010. Towards an improved understanding of farmers' behaviour: the integrative agentcentred (IAC) framework. Ecological Economics 69:2323-2333. http://dx.doi.org/10.1016/j.ecolecon.2010.07.023

Fulton, E. A., A. D. M. Smith, D. C. Smith, and I. E. van Putten. 2011. Human behaviour: the key source of uncertainty in fisheries management. Fish and Fisheries 12:2-17. http://dx.doi. org/10.1111/j.1467-2979.2010.00371.X

Grimm, V., U. Berger, F. Bastiansen, S. Eliassen, V. Ginot, J. Giske, J. Goss-Custard, T. Grand, S. K. Heinz, G. Huse, A. Huth, J. U. Jepsen, C. Jørgensen, W. M. Mooij, B. Müller, G. Pe'er, C. Piou, S. F. Railsback, A. M. Robbins, M. M. Robbins, E. Rossmanith, N. Rüger, E. Strand, S. Souissi, R. A. Stillman, R. Vabø, U. Visser, and D. L. DeAngelis. 2006. A standard protocol for describing individual-based and agent-based models. Ecological Modelling 198:115-126. http://dx.doi.org/10.1016/j.ecolmodel.2006.04.023

Hare, M., and C. Pahl-Wostl. 2001. Model uncertainty derived from choice of agent rationality: a lesson for policy assessment modelling. Pages 854-859 in N. Giambiasi and C. Frydman, editors. Simulation in industry: 13th European Simulation Symposium. The Society for Modeling and Simulation International (SCS), SCS Publications, Ghent, Belgium.

Hilborn, R., and C. J. Walters. 1992. Quantitative fisheries stock assessment: choice, dynamics and uncertainty. Chapman and Hall, New York, New York, USA.

Hinkel, J., P. Bots, and M. Schlüter. 2014. Enhancing the Ostrom social-ecological systems framework through formalization. Ecology and Society, in press.

Holling, C. S., and L. H. Gunderson. 2002. Resilience and adaptive cycles. Pages 25-62 in L. H. Gunderson and C. S. Holling, editors. Panarchy: understanding transformations in human and natural systems. Island Press, Washington, D.C., USA.

Horan, R. D., E. P. Fenichel, K. L. S. Drury, and D. M. Lodge. 2011. Managing ecological thresholds in coupled environmentalhuman systems. Proceedings of the National Academy of Sciences of the United States of America 108:7333-7338. http://dx.doi. org/10.1073/pnas.1005431108

Hunt, L. M. 2005. Recreational fishing site choice models: insights and future opportunities. Human Dimensions of Wildlife 10:153-172. http://dx.doi.org/10.1080/10871200591003409
Jakeman, A. J., R. A. Letcher, and J. P. Norton. 2006. Ten iterative steps in development and evaluation of environmental models. Environmental Modelling \& Software 21:602-614. http://dx.doi. org/10.1016/j.envsoft.2006.01.004

Jeffrey, P., and B. S. McIntosh. 2006. Description, diagnosis, prescription: a critique of the application of co-evolutionary models to natural resource management. Environmental Conservation 33:281-293. http://dx.doi.org/10.1017/S0376892906003444

Johnston, F. D., R. Arlinghaus, and U. Dieckmann. 2010. Diversity and complexity of angler behaviour drive socially optimal input and output regulations in a bioeconomic recreational-fisheries model. Canadian Journal of Fisheries and Aquatic Sciences 67:1507-1531. http://dx.doi.org/10.1139/ F10-046

Laikre, L., M. K. Schwartz, R. S. Waples, N. Ryman, and The GeM Working Group. 2010. Compromising genetic diversity in the wild: unmonitored large-scale release of plants and animals. Trends in Ecology \& Evolution 25:520-529. http://dx.doi. org/10.1016/j.tree.2010.06.013

Lorenzen, K. 2000. Allometry of natural mortality as a basis for assessing optimal release size in fish-stocking programmes. Canadian Journal of Fisheries and Aquatic Sciences 57:2374-2381. http://dx.doi.org/10.1139/f00-215

Lorenzen, K. 2005. Population dynamics and potential of fisheries stock enhancement: practical theory for assessment and policy analysis. Philosophical Transactions of the Royal Society B: Biological Sciences 360:171-189. http://dx.doi.org/10.1098/ rstb.2004.1570

Lorenzen, K., and K. Enberg. 2002. Density-dependent growth as a key mechanism in the regulation of fish populations: evidence from among-population comparisons. Proceedings of the Royal Society B: Biological Sciences 269:49-54. http://dx.doi. org/10.1098/rspb.2001.1853

Lorenzen, K., K. M. Leber, and H. L. Blankenship. 2010. Responsible approach to marine stock enhancement: an update. Reviews in Fisheries Science 18:189-210. http://dx.doi. org/10.1080/10641262.2010.491564

Mace, P. M. 1994. Relationships between common biological reference points used as thresholds and targets of fisheries management strategies. Canadian Journal of Fisheries and Aquatic Sciences 51:110-122. http://dx.doi.org/10.1139/f94-013

McGinnis, M., and E. Ostrom. 2014. SES framework: initial changes and continuing challenges. Ecology and Society, in press.

Müller, B., F. Bohn, G. Dreßler, J. Groeneveld, C. Klassert, R. Martin, M. Schlüter, J. Schulze, H. Weise, and N. Schwarz. 2013. Describing human decisions in agent-based models - ODD+D, an extension of the ODD protocol. Journal of Environmental Modelling \& Software 48:37-48. http://dx.doi.org/10.1016/j. envsoft.2013.06.003

Myers, R. A., and N. G. Cadigan. 1993. Is juvenile natural mortality in marine demersal fish variable? Canadian Journal of Fisheries and Aquatic Sciences 50:1591-1598. http://dx.doi. org/10.1139/f93-180 
Norgaard, R. B. 1994. Development betrayed: the end of progress and a coevolutionary revisioning of the future. Routledge, London, UK.

Ostrom, E. 1999. Institutional rational choice: an assessment of the institutional analysis and development framework. Pages 35-71 in $\mathrm{P}$. Sabatier, editor. Theories of the policy process. Westview, Boulder, Colorado, USA.

Ostrom, E. 2005. Understanding institutional diversity. Princeton University Press, Princeton, New Jersey, USA.

Ostrom, E. 2007. A diagnostic approach for going beyond panaceas. Proceedings of the National Academy of Sciences 104:15181-15187. http://dx.doi.org/10.1073/pnas.0702288104

Ostrom, E. 2009. A general framework for analyzing sustainability of social-ecological systems. Science 325:419-422. http://dx.doi.org/10.1126/science.1172133

Parker, D. C., B. Entwisle, R. R. Rindfuss, L. K. Vanwey, S. M. Manson, E. Moran, L. An, P. Deadman, T. P. Evans, M. Linderman, S. M. Mussavi Rizi, and G. Malanson. 2008. Case studies, cross-site comparisons, and the challenge of generalization: comparing agent-based models of land-use change in frontier regions. Journal of Land Use Science 3:41-72. http://dx.doi.org/10.1080/17474230802048151

Polhill, J. G., and N. M.Gotts. 2009. Ontologies for transparent integrated human-natural system modelling. Landscape Ecology 24:1255-1267. http://dx.doi.org/10.1007/s10980-009-9381-5

Rammel, C., S. Stagl, and H. Wilfing. 2007. Managing complex adaptive systems - a co-evolutionary perspective on natural resource management. Ecological Economics 63:9-21. http://dx. doi.org/10.1016/j.ecolecon.2006.12.014

Ricker, W. E. 1954. Stock and recruitment. Journal of the Fisheries Research Board of Canada 11:559-623. http://dx.doi.org/10.1139/ f54-039

Rogers, M. W., M. S. Allen, P. Brown, T. Hunt, W. Fulton, and B. A. Ingram. 2010. A simulation model to explore the relative value of stock enhancement versus harvest regulations for fishery sustainability. Ecological Modelling 221:919-926. http://dx.doi. org/10.1016/j.ecolmodel.2009.12.016

Rothenberg, J. 1989. The nature of modeling. Pages 75-92 in L. E. Widman, K. A. Loparo, and N. R. Nielsen, editors. Artifical intelligence, simulation, and modeling. John Wiley \& Sons, New York, New York, USA. [online] URL: http://www.rand.org/ content/dam/rand/pubs/notes/2007/N3027.pdf

Royce, W. F. 1983. Trends in fishery science. Fisheries (Bethesda) 8:10-13.

Scheffer, M., J. Bascompte, W. A. Brock, V. Brovkin, S. R. Carpenter, V. Dakos, H. Held, E. H. van Nes, M. Rietkerk, and G. Sugihara. 2009. Early-warning signals for critical transitions. Nature 461:53-59. http://dx.doi.org/10.1038/nature08227

Schlüter M., R. R. J. McAllister, R. Arlinghaus, N. Bunnefeld, K. Eisenack, F. Hölker, E. J. Milner-Gulland, B. Müller, E. Nicholson, M. Quaas, and M. Stöven. 2012. New horizons for managing the environment: a review of coupled social-ecological systems modelling. Natural Resource Modeling 25:219-272. http:// dx.doi.org/10.1111/j.1939-7445.2011.00108.x

Schmolke, A., P. Thorbek, D. L. DeAngelis, and V. Grimm. 2010. Ecological models supporting environmental decision making: a strategy for the future. Trends in Ecology \& Evolution 25:479-486. http://dx.doi.org/10.1016/j.tree.2010.05.001

Simon, H. A. 1957. Models of man. John Wiley, New York, New York, USA.

Smith, M. D., and J. E. Wilen. 2003. Economic impacts of marine reserves: the importance of spatial behavior. Journal of Environmental Economics and Management 46:183-206. http://dx. doi.org/10.1016/S0095-0696(03)00024-X

Sutton R. S., and A. G. Barto. 1998. Reinforcement learning: an introduction. MIT Press, Cambridge, Massachusetts, USA.

van Poorten, B. T., R. Arlinghaus, K. Daedlow, and S. S. HaertelBorer. 2011. Social-ecological interactions, management panaceas, and the future of wild fish populations. Proceedings of the National Academy of Sciences 108:12554-12559. http://dx.doi. org/10.1073/pnas.1013919108

Wootton, R. J. 1998. Ecology of teleost fishes. Fish and Fisheries Series 24. Kluwer Academic, Dordrecht, The Netherlands. http:// dx.doi.org/10.1007/978-94-009-0829-1 


\section{Appendix 1}

The formalized Ostrom SES framework (SESF)

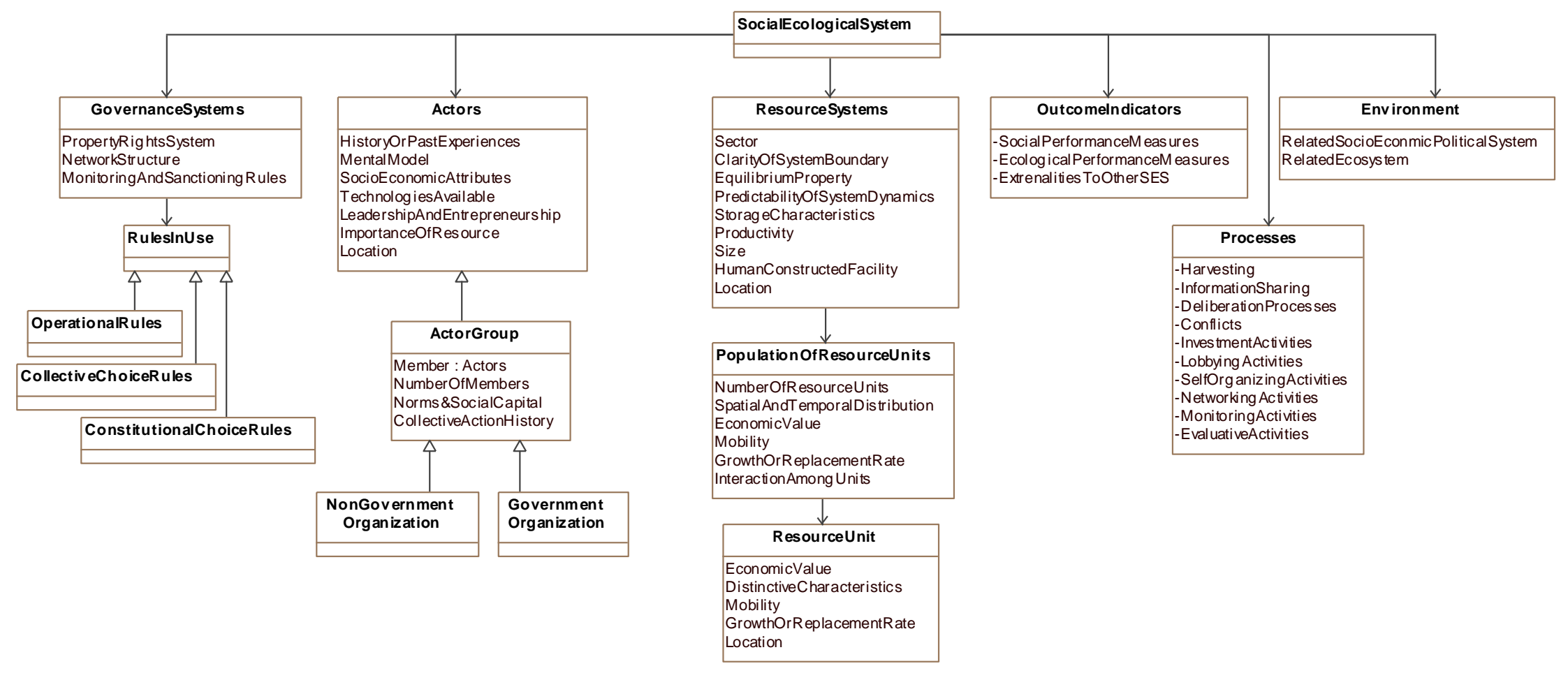

Figure A1: The SES framework (McGinnis and Ostrom 2014) in UML (Unified Modelling Language) including semantic relationships (attribution and subsumption, see Hinkel et al. (2014)) to make explicit relations between variables. Downward arrows represent attribution relationships where a variable is attributed to a concept, upward arrows represent subsumption relationships where sub-concepts are a type of the more general concept, e.g., an actor group is a type of actor. 


\section{Appendix 2}

Stocking of different sizes of fish (larvae, juvenile, recruits) with stocking density dependent on angler satisfaction.

a)

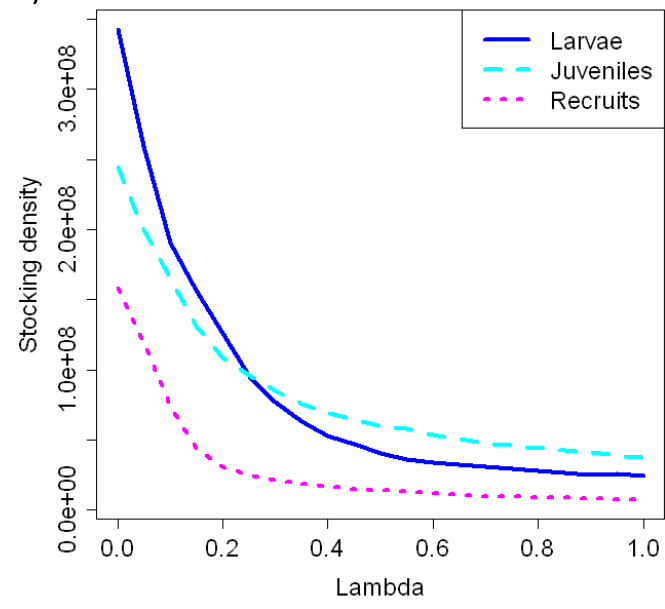

b)

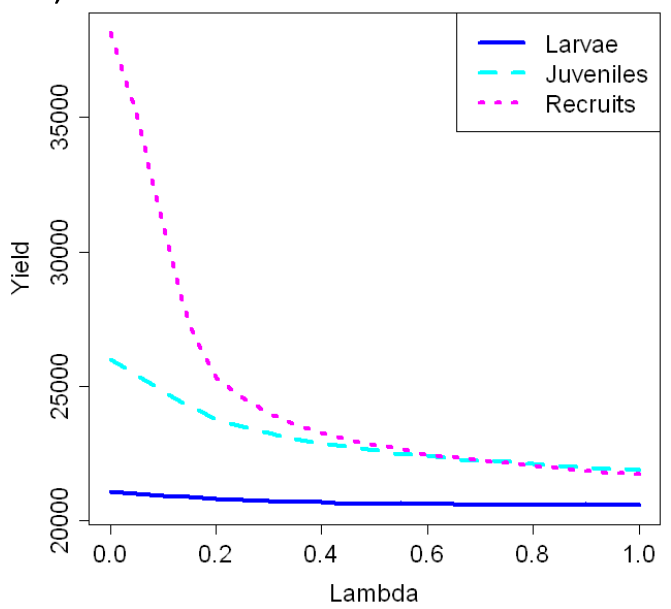

Figure A2.1: a) Stocking density and b) yields for stocking of different size of fish when stocking density is determined by angler satisfaction with varying forgetting rates lambda (low lamda equals a high memory of past experiences, high lambda represent high forgetting). When memories of past experiences the request for stocking and hences stocking densities are high, leading to higher yields. Stocking density is highest when stocking larvae.

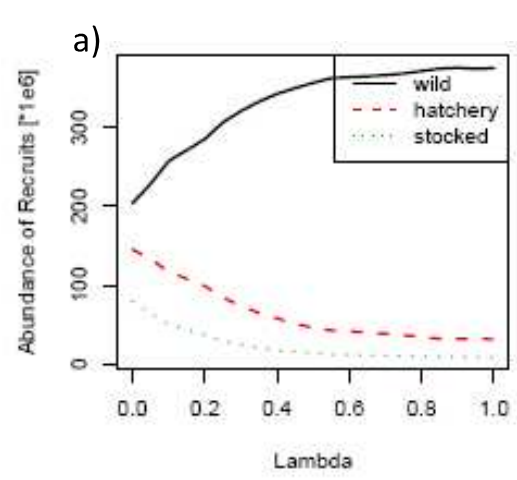

b)

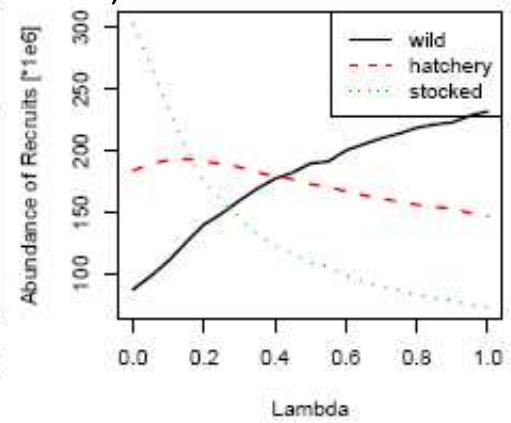

c)

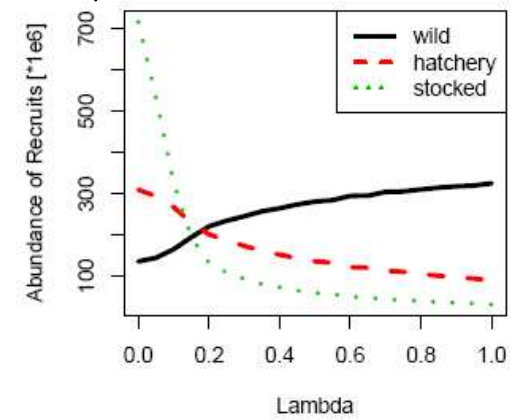

A2.2: Effects of stocking different sizes of stocked fish at densities that are determined by angler satisfaction with different forgetting rates lambda. A) stocking of larvae, b) stocking of juveniles, c) stocking of recruits. When stocking larvae no replacement takes place no matter how high the memory of anglers of past experiences. When stocking juveniles replacement can take place when anglers remember well past experience, i.e. forgetting is low. In this scenario replacement of wild fish by hatchery origin or stocked fish happens already at intermediate forgetting rates. When recruited fish are stocked replacement happens only when forgetting rates are very low. 